\title{
Guía Práctica Clínica: Guía para el abordaje del paciente adulto con mareo (primera parte)
}

Clinical practice guideline: Dizziness in adults

Andrés Pichón Riviere* y Guillermo Videla**

\begin{abstract}
Resumen
La mayoría de los individuos puede ser clasificado en alguno de los siguientes cuatro síndromes: 1) un cuadro inespecífico asociado a trastornos de ansiedad o del estado de ánimo; 2) vértigo, generalmente benigno y autolimitado; 3) desequilibrio, generalmente ancianos con déficit sensorial múltiple; 4) mareo consecutivo a un cuadro pre-sincopal. En este artículo se describe la evaluación diagnóstica del paciente adulto con mareos no vertiginosos. Una segunda entrega se explayará en manejo del subgrupo de pacientes con vértigo.
\end{abstract}

\section{Abstract}

Most individuals may be classified in one of the following four syndromes: 1) nonspecific dizziness associated with anxiety and mood disorders; 2) true vertigo, generally benign and self limited; 3) imbalance, often present in old individuals with multiple sensorial deficits; and 4) presincopal events. This article describes the diagnostic evaluation of the dizzy adult patient without vertigo. A second part of this article will be focused in vertigo patient subgroup.

Palabras clave: mareos, vertigo, evaluación diagnóstica. Key words: dizziness, vertigo, diagnosis.

Pichón Riviere A y Videla G. Guía para el abordaje del paciente adulto con mareo sin vértigo. Evid. actual. práct. ambul; 10(2): 58-60, mar-abr.2007.

\section{Introducción}

Las consultas por mareo son muy frecuentes en adultos y en cerca de $80 \%$ de los casos pueden resolverse en el ámbito de la atención primaria 1 . Sin embargo, siempre constituyen un desafío para el médico tratante.

La evaluación de estos pacientes suele ser incómoda ya que los síntomas suelen ser vagos y, muchas veces, es imposible confirmar objetivamente un diagnóstico ${ }^{2}$. Por otra parte, los pacientes suelen estar preocupados por la posible existencia de una enfermedad neurológica seria. Todo esto hace que, con frecuencia, muchos sean innecesariamente sometidos a estudios complejos y costosos o derivados prematuramente al especialista.

Casi todos los pacientes que consultan por mareo tienen entidades leves y autolimitadas, mientras que otros tienen cuadros de ansiedad o depresión en los que el mareo es un síntoma más ${ }^{3}$. Ver tablas 1 y 2 . Generalmente, el interrogatorio y el examen físico son suficientes para identificar a los individuos con enfermedades potencialmente serias.

Tabla 1: distribución de los mecanismos responsables de mareo según el ámbito de la atención de los pacientes (12 estudios que incluyeron un total de 4.536 pacientes).

\begin{tabular}{|c|c|c|c|c|c|c|}
\hline \multirow{2}{*}{\multicolumn{2}{|c|}{$\begin{array}{l}\text { Ambito y número } \\
\text { de estudios }\end{array}$}} & \multicolumn{2}{|c|}{ Vértigo } & \multicolumn{2}{|c|}{ Mareo no vertiginoso } & \\
\hline & & Periférico & Central & $\begin{array}{c}\text { No } \\
\text { específico o } \\
\text { "psiquiátrico" }\end{array}$ & \begin{tabular}{|c|} 
No \\
"psiquiátrico" \\
(incluye \\
desequilibrio y \\
presíncope)
\end{tabular} & $\begin{array}{c}\text { Causa } \\
\text { indeterminada }\end{array}$ \\
\hline Atención primaria & 2 & $43 \%$ & $9 \%$ & $21 \%$ & $34 \%$ & $4 \%$ \\
\hline Sala de emergencias & 5 & $34 \%$ & $6 \%$ & $9 \%$ & $37 \%$ & $19 \%$ \\
\hline $\begin{array}{l}\text { Clínica de vértigo o de } \\
\text { neurología }\end{array}$ & 6 & $46-49 \%$ & $7-19 \%$ & $10-20 \%$ & $17-20 \%$ & $10-18 \%$ \\
\hline
\end{tabular}

Modificado de: Kroenke K. South Med J 93 (2), 2000. Southern Medical Association
Tabla 2: frecuencia de causas específicas de mareos en los 12 estudios. Modificado de: Kroenke K. South Med J 93 (2), 2000. Southern Medical Association.

\begin{tabular}{|c|c|c|c|}
\hline & \multicolumn{2}{|r|}{ Causa } & $\begin{array}{c}\text { Media ajustada } \\
\text { por calidad }\end{array}$ \\
\hline \multirow[t]{7}{*}{ Vestibular } & \multirow[t]{4}{*}{ Periférico } & Vértigo posicional benigno & $16 \%$ \\
\hline & & Laberintitis & $9 \%$ \\
\hline & & Enfermedad de Meniere & $5 \%$ \\
\hline & & Otras & $14 \%$ \\
\hline & \multirow[t]{3}{*}{ Central } & Cerebrovascular & $65 \%$ \\
\hline & & Tumor & menor a $1 \%$ \\
\hline & & Otras & $3 \%$ \\
\hline \multirow{2}{*}{\multicolumn{2}{|c|}{ Psiquiátrico }} & Trastorno psiquiátrico & $11 \%$ \\
\hline & & Hiperventilación & $5 \%$ \\
\hline \multirow{4}{*}{\multicolumn{2}{|c|}{$\begin{array}{l}\text { No vestibular y no } \\
\text { psiquiátrico }\end{array}$}} & Pre-síncope & $6 \%$ \\
\hline & & Des equilibrio & $5 \%$ \\
\hline & & Otros & $13 \%$ \\
\hline & & Desconocida & $13 \%$ \\
\hline
\end{tabular}

* Las unidades de análisis fueron los estudios y la media fue ajustada sobre la base de los siguientes criterios metodológicos de buena calidad: aleatorización del muestreo y selección prospectiva de los participantes, protocolo estandarizado de evaluación, tipo de evaluación de la función vestibular (examen físico, exámenes complementarios, etc.) evaluación psiquiátrica, criterios explícitos de diagnóstico, cantidad de evaluadores que establecieron el diagnóstico. Los porcentajes pueden sumar más de $100 \%$ ya que en algunos pacientes el mareo fue atribuido a más de una causa.

\section{Categorización sindromática}

El término "mareo" es utilizado de manera muy diferente por cada persona y puede describir síntomas muy variados ${ }^{4}$. Una buena forma de comenzar la categorización sindromática es solicitarle que describa su "mareo" sin utilizar esta palabra en el relato. Algunos relatarán una sensación de incertidumbre

* Médico Especialista en Medicina Familiar y Master en Ciencias Epidemiológicas. Director Ejecutivo del Instituto de Efectividad Clínica y Sanitaria. apichon@iecs.org.ar

${ }_{* \star}$ Médico Especialista en Neurología. Servicio de Neurología del Hospital Italiano de Buenos Aires. guillermo.videla@hospitalitaliano.org.ar 
acerca de la propia posición o de movimiento en relación con el ambiente; otros como inestabilidad, visión borrosa, cansancio, decaimiento, humor disfórico, dolor de cabeza u otros estados subjetivos; otros que sienten como si caminaran sobre algodones, como si estuviesen borrachos o como si él o las cosas girasen o se moviesen.

Cada uno de los síndromes tiene características y etiologías propias, por lo que la forma de evaluar al paciente será muy diferente en cada uno de ellos. Para eso, es necesario pedirle que utilice términos lo más específicos posibles, que describa un episodio reciente o hacerle preguntas dirigidas para ayudarlo. Algunas se describen en el cuadro 1.

Cuadro 1: preguntas que debemos hacernos ante un paciente con mareo y algunas formas dirigidas de hacerlas.

¿Presenta vértigo verdadero?: ¿Las cosas "dan vueltas" alrededor del paciente?

¿Cómo fue el inicio de esta molestia? ¿Cuánto duran los síntomas? ¿Fue un episodio único, varios episodios seguidos o lo tiene todo el tiempo desde la fecha que usted recuerda que comenzaron los síntomas?

¿Está acompañado de síntomas auditivos, neurológicos u otros?: ¿Siente zumbidos en el oído?

¿Cuáles son los antecedentes del paciente?

¿Qué medicación toma?

En líneas generales, el paciente que consulta por mareo puede ubicarse en alguna de las categorías sindromáticas ${ }^{5,6}$ que se describen a continuación:

\section{Vértigo verdadero}

Falsa sensación de movimiento de uno mismo (vértigo subjetivo) o del ambiente (vértigo objetivo). Aproximadamente $25 \%$ de los pacientes con mareos presenta vértigo verdadero ${ }^{7,8}$. Su origen está a nivel del sistema vestibular, desde el laberinto hasta los núcleos vestibulares en el tronco cerebral. Las preguntas dirigidas en los casos que el paciente no pueda describir su problema son: ¿Tiene sensación de movimiento o de rotación de su cabeza o de su cuerpo? ¿Siente que usted da vueltas o las cosas dan vueltas alrededor de usted?

\section{Inestabilidad o desequilibrio}

Es el trastorno más común en los pacientes mayores ${ }^{5}$. Es inespecífico y generalmente multifactorial. No es un cuadro agudo y lo que predomina es la sensación de inseguridad en la bipedestación o durante la marcha y el temor a una posible caída. Suele ser debido a un déficit sensorial múltiple. Por ejemplo, pacientes diabéticos que presentan una alteración de la propiocepción debida a su neuropatía, compromiso de la visión por cataratas y alguna disfunción cerebral. El tratamiento en estos casos debe ser dirigido a mejorar cada uno de los sistemas afectados. Por ejemplo, corrección de las cataratas, rehabilitación de la marcha y de los grupos musculares del tren inferior, etc. Las preguntas dirigidas, cuando el paciente no puede expresarse correctamente, podrían ser: ¿tiene una sensación de ligereza, movilidad o inestabilidad en sus pies? ¿Se encuentra inseguro y piensa que puede caer? ¿al caminar siente adecuadamente el suelo (irregular, firme, blando, etc.)?

\section{Síncope o presíncope}

Pérdida súbita y transitoria del conocimiento o sensación de desvanecimiento inminente consecutivo a una inadecuada perfusión cerebral: hipotensión ortostática, presíncope cardíaco, arritmias, síncope vasovagal o neurogénico. En un interrogatorio dirigido las preguntas deberían ser: ¿Al levantarse bruscamente de la cama después de estar un tiempo acostado, tiene sensación de que va a desvanecerse? ¿Se le oscurece la visión?

\section{Síntomas inespecíficos y sensación de cabeza vacía}

Suelen ser los más frustrantes para el médico, ya que este síndrome engloba todos los cuadros que no pudieron agruparse en los tres anteriores. Predomina en pacientes más jóvenes en los que el mareo es un síntoma acompañante de un cuadro psiquiátrico (crisis de ansiedad, depresión, etc.). Pueden describirlo como una sensación de debilidad física, sensación de cabeza vacía o de caminar sobre algodones. El examen de estos pacientes no demuestra alteraciones significativas en el examen físico ni en la estabilidad.

\section{Evaluación y manejo del paciente con mareo sin vértigo}

\section{Paciente con presíncope}

Cuando un paciente consulta por mareo, es importante que el médico pueda discriminar si el síntoma corresponde a un presíncope. El presíncope (pródromo de síncope pero sin llegar a perder el conocimiento) puede ser relatado por el paciente como un mareo. En principio, las entidades que causan síncope y que ponen en peligro la vida (como las arritmias) no tienen pródromos (presíncope). Por lo tanto, si un paciente refiere mareo y el médico considera que es un presíncope, puede tranquilizar al paciente en cuanto a que se trata de un cuadro benigno (habitualmente de origen neurocardiogénico o secundario a hipotensión ortostática). Igualmente, el médico debe tener un alto nivel de alerta cuando considera que el mareo es secundario a un cuadro presincopal y tener en cuenta los distintos diagnósticos diferenciales.

\section{Paciente con desequilibrio}

En algunos pacientes con mareo persistente no es posible clasificar el mecanismo del síntoma como debido a vértigo o a cuadros sincopales o presincopales. Muchos de estos pacientes refieren un desequilibrio o una sensación de falta de estabilidad debida, en la mayoría de los casos, a un déficit sensorial múltiple, frecuente en ancianos, debilitados (alcohólicos crónicos, por ejemplo) o diabéticos de larga data.

El desequilibrio también puede ser secundario a problemas como ataxia cerebelosa, déficit parciales visuales, auditivos o propioceptivos, dolor articular o debilidad en los miembros inferiores como secuela de un accidente cerebrovascular, una fractura secundaria a artrosis o al reposo prolongado en cama. El inicio reciente de una medicación, especialmente sedante, o alguna enfermedad progresiva del sistema nervioso central, como la enfermedad de Parkinson, la hidrocefalia normotensiva o tumores pontocerebelosos también pueden ocasionar desequilibrio.

En pacientes con enfermedad cardiovascular o en tratamiento antihipertensivo, la hipotensión ortostática puede ser la causa de los síntomas o contribuir a agravar el cuadro. El paciente, en general, tiene pocas dificultades mientras permanece sentado, pero éstas empeoran cuando se pone de pie, camina o gira sobre si mismo. Es frecuente que describa que la sensación de inestabilidad está más relacionada con los pies que con la cabeza y que la misma se agrava con la oscuridad (porque se pierden las referencias visuales).

Es importante corregir los déficits visuales y auditivos/vestibulares, considerar la ayuda de un fisioterapeuta para mejorar la fuerza muscular o los problemas articulares y suspender o 
cambiar las drogas que puedan empeorar el cuadro (benzodiazepinas, antidepresivos, antihipertensivos). Cuando no se encuentre una explicación clara para un síntoma progresivo (ej. ataxia cerebelosa en paciente previamente sano) será necesaria la interconsulta con el especialista.

\section{Paciente con síntomas inespecíficos}

Con frecuencia los síntomas son tan inespecíficos que no es posible asignarlos a ninguna de las categorías anteriores. Se trata, en general, de pacientes jóvenes, en los que el mareo es un síntoma más de un cuadro de ansiedad o depresión. Pueden describirlo como una sensación de debilidad o inestabilidad, sensación de cabeza liviana o de caminar sobre algodones. Una frase habitual de estos pacientes es: "Doctor, no soy yo". La descripción del síntoma suele ser muy vaga y éste no compromete estado general. Un examen físico (presión arterial acostado y de pie, examen cardiovascular y neurológico) normal prácticamente descarta una causa orgánica seria, orientando el diagnóstico hacia problemas emocionales o psiquiátricos.

Muchas veces, el mareo es un componente más de un cuadro de cansancio. El cansancio o fatiga es un síntoma muy vago, cuya evaluación resulta frustrante en la mayoría de los casos. Los pacientes, generalmente, refieren falta de energía, apatía, desinterés generalizado por el trabajo, la familia o las actividades recreativas. El cansancio es un síntoma somático (equivalente) de la depresión, habitualmente acompañado de disturbios del apetito, del sueño y quejas corporales múltiples. Es importante descartar alcoholismo, co-alcoholismo, violencia, abuso sexual y abuso de drogas.

La consulta por mareo, cansancio y síntomas inespecíficos es muy frecuente en la atención ambulatoria de los adultos. En general, el manejo de estos pacientes es difícil y frustrante y el éxito terapéutico dependerá, en la mayoría de los casos, de una buena relación médico paciente. Usualmente, un interrogatorio detallado sobre los síntomas y un examen físico dedicado, permitirán descartar las patologías serias y servirán para calmar al paciente. Citar al paciente a una nueva consulta para el control de la evolución muchas veces suele dar el tiempo necesario para observar la evolución del cuadro, pero sobre todo para comenzar a conversar con el paciente sobre otros aspectos de su vida. Los "vasodilatadores cerebrales", "fortificantes" y "energizantes" que se utilizan en forma empírica para tratar el mareo son muy caras y no hay estudios serios que hayan demostrado su eficacia.

En muchos casos, los pacientes adjudican su mareo a la contractura cervical. Si bien está discutido la existencia de un mareo de origen osteoarticular cervical, ha sido claramente comprobado que los pacientes mareados generan una contractura cervical importante para limitar los movimientos cefálicos que le provocan mayor malestar. Es decir, que los trastornos cervicales son en general una consecuencia del problema más que una causa. De todas maneras deben ser tratados con fisioterapia y ejercicios.

Es muy común que a los pacientes que refieren cuadros inespecíficos cargados de angustia y sintomatología propia de la depresión se les atribuya un origen psiquiátrico a todo su problema, pero debemos tener en mente que muchos casos cursan un cuadro reactivo al vértigo o al mareo; o sea que su origen es claramente orgánico. Debemos indagar profundamente sobre el inicio de sus síntomas y no dejar de evaluar globalmente al paciente, ya que además del tratamiento sintomático con antidepresivos o ansiolíticos podría requerir medidas específicas para solucionar su problema de base.

En el cuadro 2 se describe una guía de abordaje práctico propuesta por Kroenke y col.

Cuadro 2: guía práctica para orientarse en la evaluación del paciente con mareos.

\section{Interrogatorio}

- Clarificar la sensación subjetiva evitando el uso del término mareos.

- Evaluar si empeora al:

Mover la cabeza $\longrightarrow$ vértigo posicional benigno.

Ponerse e pie $\longrightarrow$ hipotensión ortostática.

Caminar $\longrightarrow$ desequilibrio.

- Investigar síntomas asociados: síncope, nausea y/o vómitos, problemas auditivos, ataxia, déficits neurológicos focales, múltiples quejas somáticas.

- Fármacos iniciadas poco tiempo antes del comienzo de los mareos.

\section{Examen físico}

- Pulso y toma de presión arterial ortostática.

- Evaluación cardiovascular breve (soplos anomalías del ritmo). - Nistagmus y maniobras provocadoras de Hallpike/Barany (ver segunda entrega).

- Evaluación neurológica breve (déficits focales y cerebelosos)

\section{Banderas rojas para evaluar y/o derivar con urgencia}

- Síncope.

- Soplo o alteración del ritmo cardíaco.

- Nistagmus vertical o anormalidades neurológicas ? neuroimagen o interconsulta.

En la minoría de pacientes que persisten con síntomas no explicados más alla de las seis semanas puede ser necesario implementar otras pruebas diagnósticas (ej. electronistagmografía) y/o interconsultas y/o reconsiderar las causas psiquiátricas, en especial los trastornos depresivos, de ansiedad y somatomorfos.

Modificado de: Kroenke K. South Med J 93 (2), 2000. Southern Medical Association.

\section{Referencias}

1. Sloane PD, et al. Management of dizziness in primary care. J Am Board Fam Pract 1994; 7(1): 1-8.

2. Barker $L$ editor, et al. Principles of ambulatory medicine. 4th de. Baltimore: Williams \& Wilkins, 1995.

3. Kroenke K. South Med J 93(2), 2000. 2000 Southern Medical Association. Disponible en internet: http://www.medscape.com/viewarticle/410480_1

4. Goroll AH, et al. Primary care medicine: office evaluation and management of the adult patient. 3rd de. Philadelphia: Lippincott, 1995.

5. Colledge NR, et al. Evaluation of investigations to diagnose the cause of dizziness in elderly people: a community based controlled study. BMJ 1996; $313(7060)$ : 788-92.

6. Sloane PD, et al. Management of dizziness in primary care. J Am Board Fam Pract 1994; 7(1): 1-8.

7. Kroenke K, et al. Causes of persistent dizziness. A prospective study of 100 patients in ambulatory care. Ann Intern Med 1992; 117(11): 898-904

7. Kroenke K, et al. Causes of persistent dizziness. A prospective study of 100 patients in ambulatory care. Ann Intern Med 1992; 117(11): 898-904.
8. Drachman DA, et al. Benign positional vertigo: incidence and prognosis in a population-based study in Olmsted County, Minnesota. Mayo Clin Proc $1991 ; 66(6)$ : 596-601. 